\title{
Critical Period for Programming: A Never-Ending Story
}

\author{
A review of: Adair LS, Cole TJ 2003 Rapid child growth raises blood pressure in adolescent boys who were thin at \\ birth. Hypertension 4:451-456
}

A LTERATIONS OF GROWTH potential are associated with an increased risk for several chronic diseases in the adult such as: cardiovascular disease, type II diabetes and depression. This association becomes stronger when it is controlled for current body weight (1). This data implies that a fetal adaptive response to unfavorable circumstances can have enormous repercussions on public health, which is particularly relevant in developing countries that have an improved economy associated with enhanced resources to provide adequate nutrition. New information has shown that high growth velocity within the first 2 weeks of life, and not birth weight, was strongly associated with insulin resistance in adolescents born prematurely (2). Recently, Norman and Martin reported endothelial dysfunction, expressed as defects in vasodilatation, measured at three months after birth in full term small for gestational age (SGA) but not in prematurely SGA infants (3). This information suggests that the alteration of growth patterns not only expressed as birth weight but also as a transitory change in the velocity of growth in the first months of life can induce changes that will increase the risk for adult cardiovascular or metabolic disease.

Adair and Cole used data from a longitudinal cohort study of youth from the Philippines to examine the relationship between high blood pressure (BP) in adolescence and size at birth as well as patterns of growth from birth to adolescence. This cohort is extremely interesting since it gathered information on children who were growth restricted dur-

\section{Carlos E. Blanco}

ing pregnancy or in early childhood, but due to improvement in economic development in the Philippines their nutrition improved and growth velocity increased resulting in higher weight (4).

The study reported a group of 2026 adolescents with anthropometric measurements from birth to 16 years of life. The variables included: birth weight, length and gestational age. During the subsequent follow-up surveys, weight and length or height increments were measured from birth to 2 years, 2 to 8 years, 8 to 11 years in both sexes, and 11 to 15 years (in girls) and 11 to 16 years in boys. Nine groups were formed by relating birth weight and velocity of growth at different intervals (low-low, low-middle, low-high, etc). Several confounders such as: diet, physical activity, smoking habits maturation status, socioeconomic status and gestational age at birth proved not to influence the relation of body size or growth to risk of high BP.

The risk for high BP was significantly decreased for each kilogram increase in birth weight in boys when analyzed as a group. There was no significant relation of birth weight, length, body mass index (BMI), or ponderal index to risk of high $\mathrm{BP}$ in females. The risk for high BP was greatest in boys that were thin at birth, and heavy in adolescence. The group that was small at birth and presented a low BMI at adolescence did not present an increased risk for hypertension. This strongly suggests that current BMI had a determinant effect. The importance of fast growth velocity is further emphasized by examining the group of boys who were in the middle BMI group at birth, but who had faster growth rates in late childhood. In this cohort the risk for high BP was increased. Among boys in the highest third of childhood weight increments, those who were thin at birth had a predicted probability of high BP nearly twice that of those who were relatively heavy at birth $(24.5 \%$ vs $13.2 \%)$. Thus, higher childhood weight gain in the absence of fetal growth restriction was not a risk factor in this population. Males in the low-high group had significantly larger subscapular skinfolds. This finding is in keeping with previous reports associating intrauterine growth and obesity later in life (5). In general, results in girls showed absent or only minor effects of early growth.

This work offers new insights that will help to understand the mechanisms involved in the process of programming the risk for adult disease. Moreover, this data suggests that birth weight, representing the end product of intrauterine growth, is not sufficient to increase the risk for adult disease. The risk for arterial hypertension only increases when associated with enhanced weight gain velocity between 8-15 years of age in males. It could be concluded from this data that: 1) a second hit, in this case an increased growth velocity, is necessary to induce arterial hypertension and 2) the mechanisms involved in the control of arterial blood pressure can be still programmed during adolescence. Early detection of ab- 
normal cardiovascular development or its control mechanisms and modulation of growth velocity in at risk populations can be a future therapeutic possibility.

1. Barker DJP 2001 Introduction. In: Barker DJP (ed) Fetal Origins of Cardiovascular and Lung Disease. Dekker Inc, New York, vol 151, p 7

2. Singhal A, Fewtrell M Cole TJ, Lucas A 2003 Low nutrient intake and early growth for later insulin resistance in adolescents born preterm. Lancet 361:1089-1097

3. Norman M, Martin H 2003 Preterm birth attenuates association between low birth weight and endothelial dysfunction. Circulation 108:996-1001

4. Adair LS, Cole TJ 2003 Rapid child growth raises blood pressure in adolescent boys who were thin at birth. Hypertension 4:451-456

5. Vickers MH, Breier BH, Cutfield WS, Hofman PL, Gluckman PD 2000 Fetal origins of hyperphagia, obesity and hypertension and postnatal amplification by hypercaloric nutrition. Am J Physiol Endocrinol Metab 279:E83-87

Department of Pediatrics

University Hospital Maastricht

P. Debyelaan 25, P.O. Box 5800

6202 AZ Maastricht, The Netherlands

e-mail: ce.blanco@kg.unimaas.nl

DOI: 10.1203/01.PDR.0000103389.01854.D8 